\title{
Edition DETAIL
}

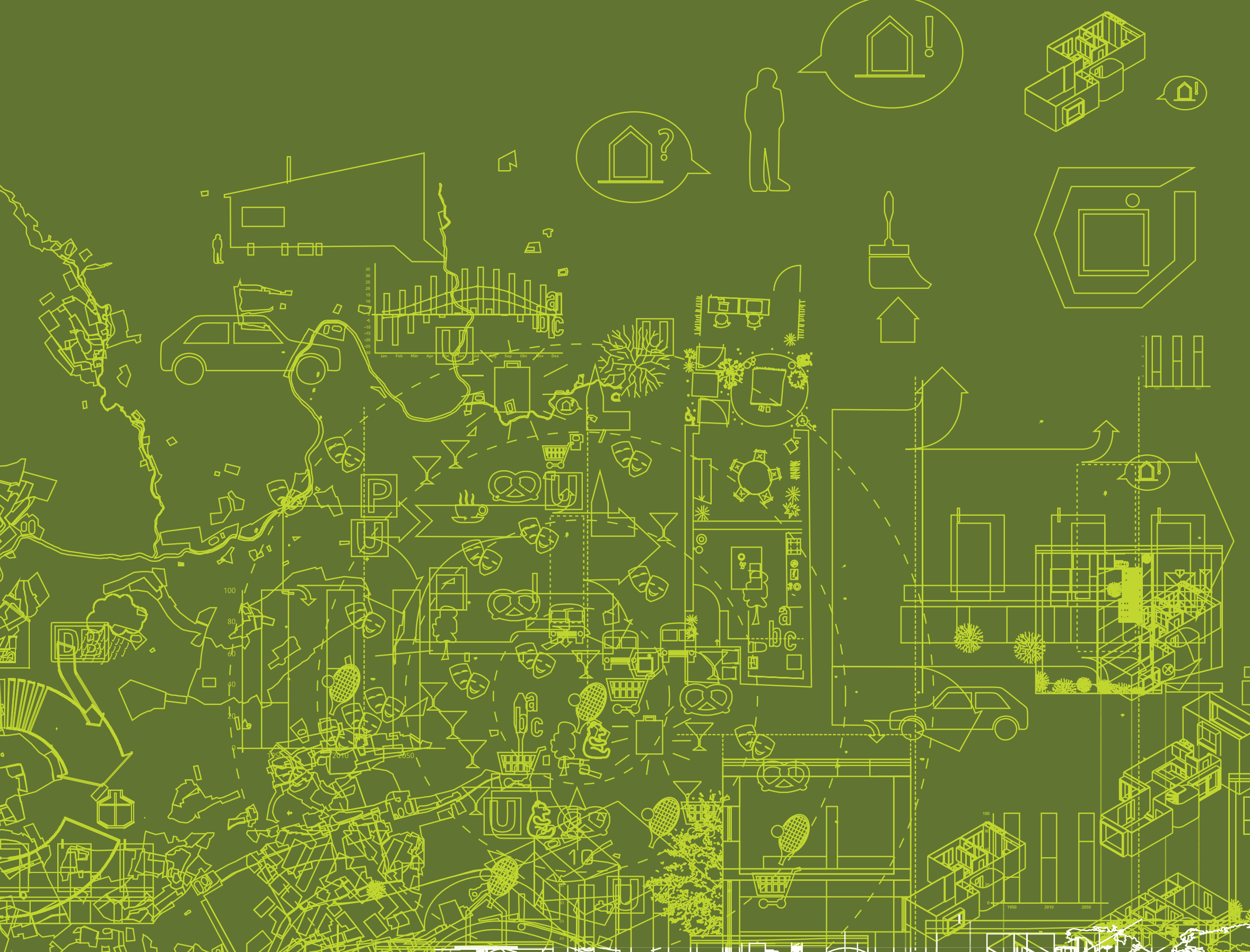

(1) 0) 1 (1)

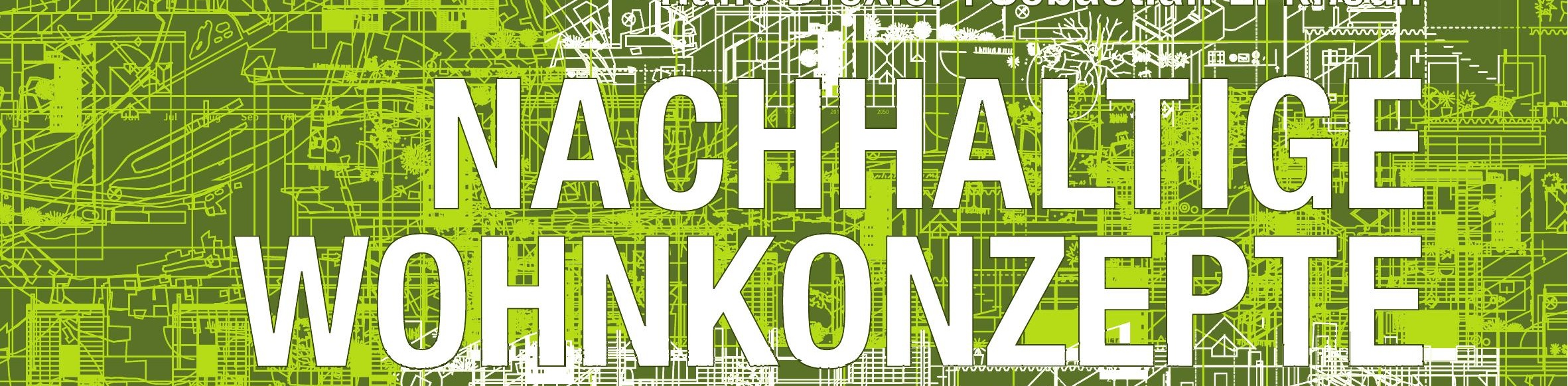
1.5.

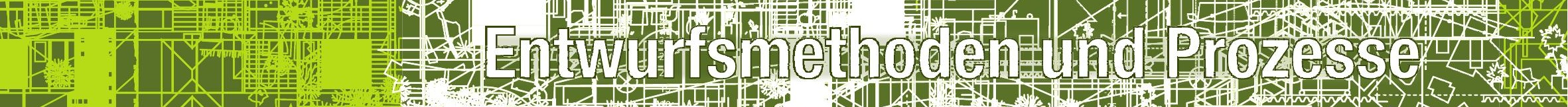
20 
NACHHALTIGE WOHNKONZEPTE 


\section{NACHHALTIGE WOHNKONZEPTE}

Entwurfsmethoden und Prozesse 


\section{IMPRESSUM}

Das Buch wurde erarbeitet am Sustainable Building Design Studio der MSA Münster

Vertretungsprofessur Dipl. Arch. ETH Hans Drexler M. Arch (Dist.)

https://www.fh-muenster.de/fb5/departments/konstruktion/drexler/Prof-Hans-Drexler.php

In Zusammenarbeit mit

Bob Gysin + Partner BGP Architekten ETH SIA BSA, Zürich

www.bgp.ch

\section{AUTOREN}

Hans Drexler

Dipl. Arch. ETH M. Arch (Dist.)

Sustainable Building Design Studio, MSA Münster

Drexler Guinand Jauslin Architekten, Frankfurt am Main - Zürich - Rotterdam

Sebastian El khouli

Dipl.-Ing. Arch. TU, Energieberater TU Darmstadt

Bob Gysin + Partner BGP Architekten ETH SIA BSA, Zürich

\section{ESSAYS}

Dominique Gauzin-Müller

Bob Gysin

\section{REDAKTION UND LEKTORAT}

Steffi Lenzen (Projektleitung)

Kirsten Rachowiak

GRAFIK, LAYOUT UND ZEICHNUNGEN

3 Karat, Frankfurt am Main, Dipl. Des. Nora Wirth

unter Mitarbeit von Dipl. Des. Katja Rudisch

www.3Karat.de

\section{MITARBEIT ZEICHNUNGEN}

Simon Kiefer, Stephanie Monteiro Kisslinger

\section{STUDENTISCHE MITARBEITER}

Alexandra Cornelius, Santosh Debus, Marta Hristova, Christine Kutscheid, Anna Sumik

\section{HERSTELLUNG/DTP}

Roswitha Siegler

REPRODUKTION

ludwig:media, Zell am See

\section{DRUCK UND BINDUNG}

Kessler Druck + Medien, Bobingen

Alle $\mathrm{CO}_{2}$-Emissionen, die aus für Recherche und Erarbeitung dieses Buchs unternommenen Flugreisen und Fahrten mit dem Pkw entstanden sind, wurden bei der Non-Profit-Stiftung myclimate (www.myclimate.org) kompensiert.

Bibliografische Information der Deutschen Nationalbibliothek

Die Deutsche Nationalbibliothek verzeichnet diese Publikation in der Deutschen Nationalbibliografie; detaillierte bibliografische Daten sind im Internet über http://dnb.d-nb.de abrufbar.

Dieses Werk ist urheberrechtlich geschützt. Die dadurch begründeten Rechte, insbesondere die der Übersetzung, des Nachdrucks, des Vortrags, der Entnahme von Abbildungen und Tabellen, der Funksendung, der Mikroverfilmung oder der Vervielfältigung auf anderen Wegen und der Speicherung in Datenverarbeitungsanlagen, bleiben, auch bei nur auszugsweiser Verwertung, vorbehalten. Eine Vervielfältigung dieses Werks oder von Teilen dieses Werks ist auch im Einzelfall nur in den Grenzen der gesetzlichen Bestimmungen des Urheberrechtsgesetzes in der jeweils geltenden Fassung zulässig. Sie ist grundsätzlich vergütungspflichtig. Zuwiderhandlungen unterliegen den Strafbestimmungen des Urheberrechts.

Dieses Buch ist auch in englischer Sprache erschienen (ISBN 978-3-920034-78-2).

(C) 2012 erste Auflage

Ein Fachbuch aus der Redaktion DETAI

Institut für internationale Architektur-Dokumentation GmbH \& Co. KG

Hackerbrücke 6, 80335 München

www.detail.de

Gedruckt auf 135 g BVS, holzfrei weiß matt gestrichen, FSC-zertifiziert.

ISBN 978-3-920034-77-5

Die Erarbeitung der Publikation war nur durch die Unterstützung des Sponsors möglich. Autoren und Verlag danken dem folgenden Unternehmen für die Förderung der Publikation:

VELUX GROUP 
Housing should be seen as a process and not as a product. Balkrishna Doshi 